\title{
Feed-Forward River Flow Control Using Differential Flatness
}

\author{
Florent Di Meglio*, Tarek Rabbani ${ }^{\dagger}$, Xavier Litrico ${ }^{\ddagger}$ and Alexandre Bayen ${ }^{\S}$ \\ *Corresponding author, Ecole des Mines de Paris, Paris, France \\ $\dagger^{\dagger}$ Department of Mechanical Engineering, UC Berkeley, CA, USA \\ ¥UMR G-EAU, Cemagref, 361 rue JF Breton, BP 5096, F-34196 Montpellier Cedex 5, France \\ $\S$ Department of Civil and Environmental Engineering, UC Berkeley, CA, USA
}

\begin{abstract}
This article presents two methods for open-loop river flow control, based on a classical hydraulic model (the Hayami model, a partial differential equation resulting from a simplification of the Saint-Venant equations). The proposed method shows the equivalence of differential flatness and a frequency approach to design a controller, by expressing the upstream flows as a function of the downstream flow (model inversion). The controller is represented as an infinite series and a statement about the convergence of this series is made. A comparison is made with controllers for thermal systems from a similar problem available in the literature. The numerical simulations show the application of the open-loop controller for irrigation canals modeled by the full Saint-Venant equations.
\end{abstract}

\section{INTRODUCTION}

The rarefaction of global water resources is a motivation for research on automation of management of water distribution systems. Large amounts of fresh water are lost due to poor management of open-channel systems. This article focuses on the management of such canals which are used to convey water from the resource (generally a dam located upstream) to a specific downstream location. Due to the fluctuations of water needs, water demand will change with time. This change in demand calls for the efficient operation of the open-channel systems to avoid overflows and to supply desired flow rates at pre-specified time instants.

Automation techniques based on optimization and control provide more efficient management strategies than manual techniques. They rely on flow models, in particular the SaintVenant equations [1] or simplified versions of these equations to describe one-dimensional hydraulic systems. Level regulation and control of the water flow are among the methods used to improve the efficiency of irrigation systems. These techniques allow engineers to regulate the flow in hydraulic canals and therefore to irrigate large areas according to predicted consumption.

In this article, the specific problem of controlling downstream flow in a one-dimensional hydraulic canal by upstream discharges is investigated. Several approaches to this problem have already been described in the literature. The majority of these approaches use linear controllers to control the nonlinear dynamics of the canal system. Such methods include transfer function analysis for Saint-Venant equations [14] which enables the use of classical control techniques for feedback control. Rieman invariants for hyperbolic conservation laws as in [4] can be used to construct Lyapunov functions, used for stabilization purposes. Adjoint methods [21] have been used for estimation and control, via sensitivity analysis. Open-loop control methods have been developed either by computing the solutions of the flow equations backwards using discritization and finite difference methods [3], [2], or using a finite dimensional approximation in the frequency domain [13]. Our approach is to design an open-loop controller that expresses the upstream discharges explicitly as a function of the desired downstream flow at a given location. It can be shown using Lyapunov stability method that the open-loop system is stable by [9], and [22], which provides another justification for open-loop control of the considered system. We will use two approaches: a differential flatness approach based on CauchyKovalevskaya series and a frequency approach based on the Laplace transform.

In the context of partial differential equations, differential flatness was used to investigate the related problem of heavy chains motion planning [18], as well as the Burgers equation in [17] or the telegraph equation in [7]. The theory of differential flatness, which was first developed in [6], consists in a parametrization of the trajectories of a system by one of its output which suits our problem. Using the Laplace frequency domain to derive the control was introduced by [20]. In this article, problems previously tackled by differential flatness like the heavy chains problem, and the telegraph equation [20] are solved by the frequency approach.

Starting from the classical Saint-Venant equations widely used to model unsteady flows in rivers [1], we present a model simplification and a linearization which lead to the Hayami partial differential equation as shown in [16]. The practicality of using the Hayami equation lies in the need of only two numerical parameters to characterize flow conditions: celerity and diffusivity. The original Saint-Venant equation requires the knowledge of the full geometry of the canal and of the roughness coefficient, which make it impractical for long rivers 
where these parameters are difficult to estimate.

The problem of controlling the Hayami equation was already investigated in [8] and [14] with transfer function analysis, and in [11] for parameter estimation. The Hayami equation is closely linked to the diffusive wave equation with quadratic source terms, which have been studied in [5] and [15]. The difference between our problem and the aforementioned problem is the nature of the boundary conditions: indeed, contrary to the heat equation case, one cannot impose a value for the downstream discharge. In river flow, there are hydraulic structures such as weirs which impose a static relation between water elevation and the flow. In fact, we show that the solution of our problem is a composite of the solution in [15] and an additional term which captures the boundary condition set by the hydraulic structure.

The article is structured as follows: a description of the physical problem and the system of equations to be solved is introduced first (section II). Then, in section III, a solution of these equations is devised using differential flatness and transfer function analysis. The equivalence of the two approaches is shown analytically. A statement of convergence is made about the controller infinite series. A numerical assessment of the open-loop controller is finally presented and discussed in section IV. In particular, the difference with controllers synthesized in the context of heat transfer is illustrated through numerical simulation. Applications of the controller on the fully nonlinear Saint-Venant model are presented.

\section{PhysicAl Problem}

The system of interest is a hydraulic canal of length $L$ with a prismatic or rectangular cross-section. In this section we present the equations that govern the system, the SaintVenant equations. We then derive the Hayami model which is a simplification of these equations.

\section{A. Saint-Venant Equations}

The Saint-Venant equations [1] are generally used to describe unsteady flows in rivers or canals [16]. These equations are written as follows:

$$
\begin{aligned}
A_{t}+Q_{x} & =0 \\
Q_{t}+\left(\frac{Q^{2}}{A}\right)_{x}+g A Y_{x} & =g A\left(S_{b}-S_{f}\right)
\end{aligned}
$$

with $A(x, t)$ the wetted cross-sectional area $\left(m^{2}\right), Q(x, t)$ the discharge $\left(\mathrm{m}^{3} / \mathrm{s}\right)$ across section $A(x, t), Y(x, t)$ the water depth $(m), S_{f}(x, t)$ the friction slope, $S_{b}$ the bed slope, and $g$ the gravitational acceleration $\left(\mathrm{m}^{2} / \mathrm{s}\right)$. These variables are linked by the following relations: $A(x, t)=Y(x, t) B(x, t)$, $Z(x, t)=Y(x, t)+S_{b}(L-x)$ and $Q(x, t)=V(x, t) A(x, t)$ where $Z(x, t)$ is the absolute water elevation $(m), V(x, t)$ is the mean water velocity across section $A(x, t)$, and $B(x, t)$ is the bed width. Equation (1) is referred to as the mass conservation equation, and equation (2) is called the momentum conservation equation. We assume that there is a crossstructure, at the right end of the canal, which means that there is an analytical function $W(\cdot)$ that relates $Q$ and $Z$ at $x=L$, i.e:

$$
Q(L, t)=W(Z(L, t))
$$

For a weir structure, this relation can be assumed to be $Q(L, t)=C_{w}\left(Z(L, t)-Z_{w}\right)^{3 / 2}$ where $Z_{w}$ is the weir elevation, and $C_{w}$ a positive constant depending on the physical characteristics of the weir. The Saint-Venant equations are linearized to derive the Hayami model.

\section{B. Hayami Model}

Depending on the characteristics of the river, some terms in the momentum equation (2) can be neglected, which allows us to simplify the two equations and to assemble them into a single partial differential equation. As shown in [14], assuming that the inertia terms $Q_{t}+\left(\frac{Q^{2}}{A}\right)_{x}$ can be neglected with respect to $g A Y_{x}$ will lead to the diffusive wave model:

$$
\begin{aligned}
B Y_{t}+Q_{x} & =0 \\
Z_{x} & =-S_{f}
\end{aligned}
$$

The two equations can be combined and will lead to the diffusive wave equation:

$$
Q_{t}+C Q_{x}-D Q_{x x}=0
$$

where $Q(x, t)$ is the flow $\left(m^{3} / s\right), C(Q, Z(x, t), x)$ and $D(Q, Z(x, t), x)$ usually known as the celerity and the diffusivity are non-linear functions of the flow, depth, and location [6]. Linearizing equation (4) around a reference discharge $Q_{0}$ (i.e. $\left.Q(x, t)=Q_{0}+q(x, t)\right)$ leads to the Hayami equation:

$$
q_{t}+C_{0} q_{x}-D_{0} q_{x x}=0
$$

where $q(x, t)$ is the deviation from the nominal flow $Q_{0}$, $C_{0}\left(Q_{0}\right)$ and $D_{0}\left(Q_{0}\right)$ are the nominal celerity and diffusivity. We call $Z_{0}$ and $B_{0}$ the reference elevation and width, and also assume that $Z(x, t)=Z_{0}+z(x, t)$, and $B(x, t) \sim B_{0}$, therefore equation (4) can be linearized as follows:

$$
B_{0} z_{t}+q_{x}=0
$$

where we have substituted $Y_{t}$ by $\left(Z-S_{b}(L-x)\right)_{t}=Z_{t}$ before linearizing. The right boundary condition (3) is also linearized and becomes:

$$
q(L, t)=b z(L, t)
$$

where $b$ is the linearization constant. The value of this constant depends on the weir geometry: length, height, and discharge coefficient. 


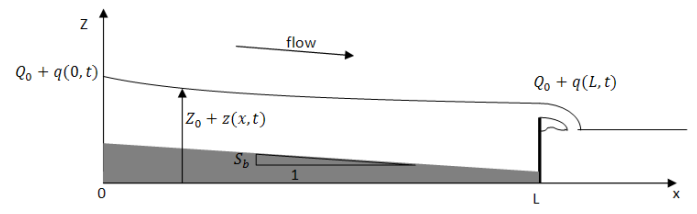

Fig. 1. Schematic representation of the canal with weir structure.

\section{Open-Loop Control Problem}

The control problem illustrated in Figure 1, consists in determining the control $u(t)=q(0, t)$, i.e. the flow of the upstream discharge, that yields the desired downstream discharge $y(t)=q(L, t)$, where $y(t)$ is a user-defined profile of flow over time at the end of the canal.

We therefore have to solve a problem whose dynamics are modeled by the following equations:

$$
\begin{aligned}
& \forall x \in[0, L] \forall t \in[0, T] \quad D_{0} q_{x x}-C_{0} q_{x}=q_{t} \\
& \forall x \in[0, L] \forall t \in[0, T] \quad B_{0} z_{t}+q_{x} \quad=0 \\
& \forall t \in[0, T] \quad q(L, t) \quad=b z(L, t)
\end{aligned}
$$

And a boundary condition is imposed at $x=L$ by equation (10):

$$
\forall t \in[0, T] q(L, t)=y(t)
$$

The appropriate control $u(\cdot)$ which will generate the $y(t)$ defined by (10) is defined by equation (11):

$$
\forall t \in[0, T] q(0, t)=u(t)
$$

\section{Computation OF THE Open Loop CONTROL InPUT FOR THE HAYAMI MODEL}

In this section we solve the control problem given by equations (7-10) and try to parametrize the flow $q(x, t)$ in terms of the discharge $q(L, t)$ or $y(t)$. We will produce a solution to this problem using two approaches:

1) An approach based on Cauchy-Kovalevskaya decomposition

2) A frequency approach based on Laplace transform.

We define the following intermediate variables: $\alpha=\frac{C_{0}}{2 D_{0}}, \beta=$ $\frac{1}{\sqrt{D_{0}}}, \kappa=\frac{B_{0}}{b} \frac{\alpha^{2}}{\beta^{2}}-\alpha$, and the function $f(t)=e^{\frac{\alpha^{2}}{\beta^{2}} t}$ which will simplify our calculations. The transfer function approach does not make the assumption that the considered functions are expandable in the Cauchy-Kovelevskaya form, therefore is a priori more general. In the present case as will be shown below, the result for the algebraic expression obtained shows equivalence of the two methods (a posteriori assessment).

\section{A. Cauchy-Kovalevskaya Form Approach}

As shown in [19], equation (7) can be transformed into the heat equation. Let us consider the following transformation:

$$
q(x, t)=h(x, t) p(x, t)
$$

where

$$
h(x, t)=e^{\left(-\frac{\alpha^{2}}{\beta^{2}} t+\alpha(x-L)\right)} .
$$

We have:

$$
\begin{aligned}
p_{t} & =e^{\left(\frac{\alpha^{2}}{\beta^{2}} t-\alpha(x-L)\right)}\left(q_{t}+\frac{\alpha^{2}}{\beta^{2}} q\right) \\
p_{x} & =e^{\left(\frac{\alpha^{2}}{\beta^{2}} t-\alpha(x-L)\right)}\left(q_{x}-\alpha q\right) \\
p_{x x} & =e^{\left(\frac{\alpha^{2}}{\beta^{2}} t-\alpha(x-L)\right)}\left(q_{x x}-2 \alpha q_{x}+\alpha^{2} q\right) .
\end{aligned}
$$

Substituting in equation (7), $p(x, t)$ will satisfy:

$$
p_{t}=\frac{1}{\beta^{2}} p_{x x}
$$

The problem (7) - (10) can now be reformulated as follows:

$$
\begin{aligned}
\forall x \in[0, L] \forall t & \in[0, T] \quad p_{t}=\frac{1}{\beta^{2}} p_{x x} \\
\forall x \in[0, L] \forall t & \in[0, T] B_{0} z_{t}=-h(x, t)\left(p_{x}+\alpha p\right) \\
\forall t & \in[0, T] p(L, t)=b e^{\left(\frac{\alpha^{2}}{\beta^{2}} t\right)} z(L, t) \\
\forall t & \in[0, T] p(L, t)=e^{\left(\frac{\alpha^{2}}{\beta^{2}} t\right)} y(t)
\end{aligned}
$$

The Cauchy-Kovalevskaya form [10] consists of expressing the solution of the PDE as a function of $p(L, t)(\operatorname{resp} . z(L, t))$ and all its derivatives. We assume the following form for $p$ and $z$ :

$$
\begin{aligned}
& p(x, t)=\sum_{i=0}^{+\infty} p_{i}(t) \frac{(x-L)^{i}}{i !} \\
& z(x, t)=\sum_{i=0}^{+\infty} z_{i}(t) \frac{(x-L)^{i}}{i !}
\end{aligned}
$$

where $p_{i}(t)$ and $z_{i}(t)$ are smooth functions. We have:

$$
\begin{aligned}
p_{t} & =\sum_{i=0}^{+\infty} \dot{p}_{i} \frac{(x-L)^{i}}{i !} \\
p_{x x} & =\sum_{i=0}^{+\infty} p_{i+2} \frac{(x-L)^{i}}{i !}
\end{aligned}
$$

After substitution in equation (15), we obtain:

$$
\sum_{i=0}^{+\infty} \dot{p}_{i} \frac{(x-L)^{i}}{i !}=\frac{1}{\beta^{2}} \sum_{i=0}^{+\infty} p_{i+2} \frac{(x-L)^{i}}{i !}
$$

Equating the coefficients of $\frac{(x-L)^{i}}{i !}$ gives for all $i \in \mathbf{N}$ :

$$
p_{i+2}(t)=\beta^{2} \dot{p}_{i}(t)
$$

Additionally, we have $p_{0}=p(L, t)$ and $z_{0}=z(L, t)$. We still need a condition on $p_{1}$ to be able to express every $p_{i}$ as a function of $p_{0}$. We combine equation (16) and equation (17) to obtain a boundary condition on $p$ at $x=L$. We have:

$$
z_{t}=\sum_{i=0}^{+\infty} \dot{z}_{i} \frac{(x-L)^{i}}{i !}
$$

So that $\dot{z}_{0}=z_{t}(L, t)$, and equation (16), with $x=L$ gives: 


$$
B_{0} \dot{z}_{0}+e^{-\frac{\alpha^{2}}{\beta^{2}} t}\left(p_{1}+\alpha p_{0}\right)=0
$$

In addition, equation (17) gives:

$$
p_{0}=b z_{0} e^{\frac{\alpha^{2}}{\beta^{2}} t}
$$

so that:

$$
\dot{z}_{0}=\frac{1}{b}\left(\dot{p}_{0}-\frac{\alpha^{2}}{\beta^{2}} p_{0}\right) e^{-\frac{\alpha^{2}}{\beta^{2}} t}
$$

and eventually:

$$
p_{1}=-\frac{B_{0}}{b} \dot{p_{0}}+\kappa p_{0}
$$

Using the induction relation (19) and the expression of $p_{0}$ and $p_{1}$, we can compute separately the odd and even terms:

$$
\begin{aligned}
p_{2 i} & =\beta^{2 i} p_{0}^{(i)} \\
p_{2 i+1} & =\kappa \beta^{2 i} p_{0}^{(i)}-\frac{B_{0}}{b} \beta^{2 i} p_{0}^{(i+1)}
\end{aligned}
$$

Therefore, we can formally write $p(x, t)$ as follows:

$$
\begin{aligned}
p(x, t)= & \sum_{i=0}^{+\infty} \beta^{2 i} p_{0}^{(i)} \frac{(x-L)^{2 i}}{(2 i) !} \\
& +\sum_{i=0}^{+\infty} \beta^{2 i}\left(\kappa p_{0}^{(i)}-\frac{B_{0}}{b} p_{0}^{(i+1)}\right) \frac{(x-L)^{2 i+1}}{(2 i+1) !}
\end{aligned}
$$

From equation (18), we deduce that $p_{0}(t)=f(t) y(t)$. The final parametrization of the flow $q(x, t)$ will have the form:

$$
q(x, t)=h(x, t)\left(T_{1}(x, t)+\kappa T_{2}(x, t)-\frac{B_{0}}{b} T_{3}(x, t)\right)
$$

where

$$
\begin{aligned}
T_{1}(x, t) & =\sum_{i=0}^{+\infty}(f y)^{(i)} \frac{\beta^{2 i}(x-L)^{2 i}}{(2 i) !}, \\
T_{2}(x, t) & =\sum_{i=0}^{+\infty}(f y)^{(i)} \frac{\beta^{2 i}(x-L)^{2 i+1}}{(2 i+1) !},
\end{aligned}
$$

and

$$
T_{3}(x, t)=\sum_{i=0}^{+\infty}(f y)^{(i+1)} \frac{\beta^{2 i}(x-L)^{2 i+1}}{(2 i+1) !} .
$$

Equation (20) relates the discharge variation $q(x, t)$ as a function of the desired output $y(t)$ which corresponds to the discharge $q(L, t)$ at the downstream end of the canal. This solution is formal, and the convergence of the infinite series needs to be assessed. Before we investigate this issue, we solve the problem in the frequency domain, to prove the equivalence of the two approaches.
B. Feed-forward Controller Design using the Frequency Approach Based on Laplace Transform

The frequency approach uses the Laplace transform of the equations of section II-C, and provides algebraic expressions instead of partial differential equations by converting derivatives w.r.t time $t$ into multiplications by the frequency $s$. After finding the algebraic expression of the solution in the Laplace domain, we can carry out an inverse Laplace transform to find the parametrization of $q(x, t)$ in terms of the desired output flow $y(t)$. For Laplace notations, we define for any function $l(x, t), t \geq 0$, the temporal Laplace transform, noted $\mathcal{L}(l(x, t))$, or $\hat{l}(x, s)$ to be: $\hat{l}(x, s)=\int_{0}^{\infty} e^{-s t} l(x, t) d t$. We start with the frequency representation of the Hayami model which is the Laplace formulation of the system (7)-(9), derived in appendix A.

$$
\hat{q}(x, s)=F(x, s) \hat{u}(s)
$$

with

$$
F(x, s)=\frac{P(x, s)}{Q(s)}
$$

where $P(x, s)$ and $Q(s)$ are defined in appendix A.

Equation (25) relates the flow in the Laplace domain at any point $x, \hat{q}(x, s)$, to the input flow prescribed by the control input $\hat{u}(s)$. We want to derive an algebraic expression that relates $\hat{q}(x, s)$ to the desired output $\hat{y}(s)$. We proceed by evaluating equation (24) at $x=L$ to obtain:

$$
\hat{y}(s)=F(L, s) \hat{u}(s)
$$

Dividing equation (24) by equation (26) leads to the following expression:

$$
\frac{\hat{q}(x, s)}{\hat{y}(s)}=\frac{P(x, s)}{P(L, s)}
$$

Developing the hyperbolic functions of $P(x, s)$, in terms of their power series, we obtain:

$$
\begin{aligned}
P(x, s)= & e^{\alpha x}\left(\left(B_{0} s+\alpha b\right) \sum_{i=0}^{+\infty} \frac{(\gamma(L-x))^{2 i+1}}{(2 i+1) !}\right. \\
& \left.+\gamma b \sum_{i=0}^{+\infty} \frac{(\gamma(L-x))^{2 i}}{(2 i) !}\right)
\end{aligned}
$$

We substitute equation (28) into equation (27) and express $\hat{q}(x, s)$ in terms of the constants defined in section III.

$$
\begin{aligned}
\hat{q}(x, s)= & e^{\alpha(x-L)}\left(\sum_{i=0}^{+\infty}\left(s+\frac{\alpha^{2}}{\beta^{2}}\right)^{i} \frac{\beta^{2 i}(x-L)^{2 i}}{(2 i) !}\right. \\
& +\sum_{i=0}^{+\infty} \beta^{2 i}\left(\kappa\left(s+\frac{\alpha^{2}}{\beta^{2}}\right)^{i}-\frac{B_{0}}{b}\left(s+\frac{\alpha^{2}}{\beta^{2}}\right)^{i+1}\right) \\
& \left.\frac{(x-L)^{2 i+1}}{(2 i+1) !}\right) \hat{y}(s)
\end{aligned}
$$

We carry out the inverse Laplace transform and express $q(x, t)$ in terms of $y(t)$ in the time domain. Using the inverse 
Laplace transformation rules: $\mathcal{L}^{-1}(\hat{g}(s+a))=g(t) e^{-a t}$ and $\mathcal{L}^{-1}(s \hat{g}(s))=\dot{g}(t)+g(0)$ where $g(t)$ is any time function and $a$ is a constant, we can use the following identity:

$$
\mathcal{L}^{-1}\left((s+a)^{i} \hat{g}(s)\right)=\left(g(t) e^{a t}\right)^{(i)}
$$

where we have assumed zero initial conditions. Applying equation (30) with $a=\frac{\alpha^{2}}{\beta^{2}}$ and $\hat{g}(s)=\hat{y}(s)$ to equation (29), we obtain:

$$
q(x, t)=h(x, t)\left(T_{1}(x, t)+\kappa T_{2}(x, t)-\frac{B_{0}}{b} T_{3}(x, t)\right)
$$

where $T_{1}(x, t), T_{2}(x, t), T_{3}(x, t)$ are defined in equations (21), (22), and (23), and $h(x, t)$ is defined in equation (13).

The algebraic solution obtained with the frequency approach is indeed identical to the solution obtained with the CauchyKovalevskaya approach.

\section{Convergence of the Infinite Series}

We now give the formal proof of convergence of the series in equation (31) or (20). We assume that the flat output $y(t)$ is a Gevrey function of order $\alpha>0$, i.e.:

$$
\exists m, l \in \mathbf{R} \exists \alpha>0 \forall n \in \mathbf{N} \sup _{t \in[0, T]}\left|y^{(n)}(t)\right|<m \frac{(n !)^{\alpha}}{l^{n}}
$$

$f(t)=e^{\frac{\alpha^{2}}{\beta^{2}} t}$ is Gevrey of order 0 , and therefore is Gevrey of order $\alpha$. The product of two Gevrey functions of same order is a Gevrey function of the same order, as a consequence, $f(t) y(t)$ is Gevrey of order $\alpha>0$. We will use the CauchyHadamard theorem [5] which states that the radius of convergence, $r$, of the Taylor series $\sum_{i=0}^{+\infty} a_{n} x^{n}$ to be $r=\frac{1}{\limsup _{n \rightarrow+\infty}\left|a_{n}\right|^{1 / n}}$. The radius of convergence for $T_{3}(x, t)$ is given by:

$$
\frac{1}{\rho}=\limsup _{i \rightarrow+\infty}\left(\frac{\beta^{2 i}\left|(f y)^{(i+1)}(t)\right|}{(2 i+1) !}\right)^{\frac{1}{2 i+1}}
$$

where $\rho$ is the radius of convergence around $L$. We can find an upper bound to $\frac{1}{\rho}$ by inducing the property of bounds on a Gevrey function of order $\alpha>0$ from equation (32).

$$
\begin{aligned}
\frac{1}{\rho} & \leq \limsup _{i \rightarrow+\infty}\left(\frac{\beta^{2 i} m \frac{((i+1) !)^{\alpha}}{l^{i+1}}}{(2 i+1) !}\right)^{\frac{1}{2 i+1}} \\
& \leq \limsup _{i \rightarrow+\infty} \frac{\beta^{\frac{2 i}{2 i+1}} m^{\frac{1}{2 i+1}}}{l^{\frac{i+1}{2 i+1}}}\left(\frac{((i+1) !)^{\alpha}}{(2 i+1) !}\right)^{\frac{1}{2 i+1}} \\
& \sim \limsup _{i \rightarrow+\infty} \frac{\beta}{\sqrt{l}} \frac{i+1}{2 i+1}\left(\frac{i+1}{e}\right)^{\frac{(\alpha-2) i+(\alpha-1)}{2 i+1}} \\
& \sim \begin{cases}+\infty & \alpha>2 \\
\frac{\beta}{2 \sqrt{l}} & \alpha=2 \\
0 & \alpha<2\end{cases}
\end{aligned}
$$

where in equation (33) we have used the fact that $((i+1) !)^{\frac{1}{i+1}} \sim \frac{i+1}{e}$, and $((2 i+1) !)^{\frac{1}{2 i+1}} \sim \frac{2 i+1}{e}$ as an immediate consequence of the Stirling formula. Also we have used $m^{\frac{1}{2 i+1}} \sim 1, \beta^{\frac{2 i}{2 i+1}} \sim \beta$ and $l^{\frac{i+1}{2 i+1}} \sim \sqrt{l}$. This will ensure an infinite radius of convergence for $\alpha<2$. Similar calculations can be held for $T_{1}(x, t)$ and $T_{2}(x, t)$ leading to the following conclusions:

- Equation (31) or (20) converges with an infinite radius of convergence for the choice of a Gevrey function $y(t)$ of order $\alpha<2$.

- For $\alpha=2$, We will have a sufficient condition on the convergence of the series if $x \in\left[0, L-\frac{2 \sqrt{l}}{\beta}\right] \cup$ $\left[L+\frac{2 \sqrt{l}}{\beta},+\infty\right)$, yet there could be convergence if $x \in$ $\left[L-\frac{2 \sqrt{l}}{\beta}, L+\frac{2 \sqrt{l}}{\beta}\right]$.

- We can draw no conclusions on the convergence of the series when $\alpha>2$.

\section{Numerical Assessment of the Performance of THE FEED-FORWARD CONTROLLER}

In this section, we compute the control command $u(t)$ by evaluating equation (20) or (31) at $x=0$. We subsequently simulate the controller numerically on the Hayami model equations (7) - (9) in order to evaluate their behavior before testing them on the Saint-Venant equations. This section successively investigates numerical simulations for the Hayami and the Saint-Venant models.

\section{A. Hayami Model Simulation}

From section III-C, the infinite series convergence is ensured by choosing $y(t)$ to be a Gevrey function of order $\alpha<2$. To meet this convergence condition following [10], we introduce the bump function $\phi_{\sigma}(t): \mathbf{R} \rightarrow \mathbf{R}$ defined as

$$
\phi_{\sigma}(t)= \begin{cases}0 & t<0 \\ \frac{\int_{0}^{t / T} \exp \left(-1 /\left((\tau(1-\tau))^{\sigma}\right) d \tau\right.}{1} & 0 \leq t \leq T \\ \int_{0}^{1} \exp \left(-1 /\left((\tau(1-\tau))^{\sigma}\right) d \tau\right. & \\ 1 & t>T\end{cases}
$$

where $\sigma>1, T>0$. The Gevrey order of the bump function is $1+1 / \sigma$. The function $\phi_{\sigma}(t)$ is used in [5], [6], [10], [15], [20], it is strictly increasing from 0 at $t=0$ to 1 at $t=T$ with zero derivatives at $t=0$ and $t=T$. The larger the $\sigma$ parameter is, the faster is the transition. Setting $y(t)=q_{1} \phi_{\sigma}(t)$ will allow us to have a transition from zero discharge flow for $t \leq 0$ to a discharge flow equal to $q_{1}$ for $t \geq T$, where $q_{1}$ is a constant.

The upstream discharges or the control input $u(t)$ can be computed by substituting $x=0$ in equation (20) or (31). We obtain:

$$
u(t)=h(0, t)\left(T_{1}(0, t)+\kappa T_{2}(0, t)-\frac{B_{0}}{b} T_{3}(0, t)\right)
$$




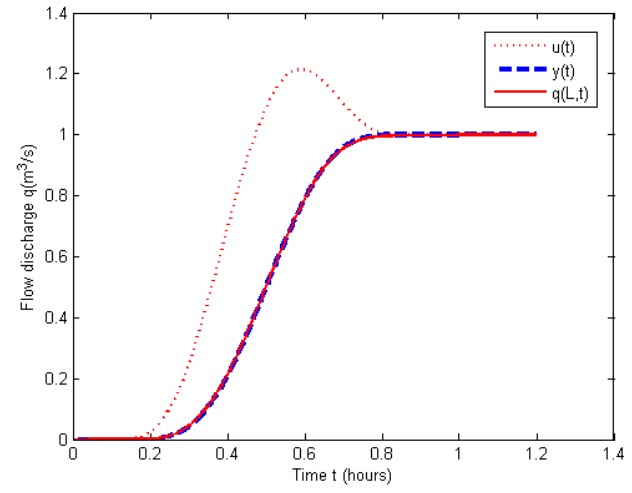

Fig. 2. Results of the numerical simulation of feed-forward control of the Hayami equation. The desired downstream discharge is $y(t)$, the upstream discharge is $u(t)$, and the downstream discharge computed by solving the Hayami model is $q(L, t)$.

For the Hayami model simulation, we will consider the transition from a downstream discharge of $0 \mathrm{~m}^{3} / \mathrm{s}$ to $1 \mathrm{~m}^{3} / \mathrm{s}$ in 1 hour ( $T=3600$ seconds). We will take $\sigma=2$ which will imply $y(t)$ to be a Gevrey-function of order 1.5 thus satisfying the convergence condition in section III-C. The model parameters are $L=1000 \mathrm{~m}, C_{0}=20 \mathrm{~m} / \mathrm{s}$, $D_{0}=1800 \mathrm{~m}^{2} / \mathrm{s}, B_{0}=7 \mathrm{~m}$, and $b=1 \mathrm{~m}^{2} / \mathrm{s}$. The infinite series of the control input $u(t)$ is approximated using 80 terms of the infinite series, and equations (7), (8), (9), and (11) are solved using the Crank-Nicholson scheme. The solution of the numerical scheme at $x=L$ or $q(L, t)$ is compared to $y(t)$, the desired downstream discharge flow. The results of this simulation are shown in figure 2.

The discharge at the downstream follows the desired discharge perfectly which validates our control input. Now we will compare our result to other problems from the literature.

In [10], an explicit open loop controller was derived for the heat equation with zero gradient boundary conditions. With some simple transformations in time and space we can relate the results [10] to our problem. The transformed version of [10] has the following form:

$$
\begin{aligned}
& \forall x \in[0, L] \forall t \in[0, T] \quad D_{0} q_{x x}-C_{0} q_{x}=q_{t} \\
& \forall t \in[0, T] \quad q_{x}(L, t) \quad=0 \\
& \forall t \in[0, T] \quad q(L, t) \quad=y(t) \\
& \forall t \in[0, T] \quad q(0, t) \quad=u(t)
\end{aligned}
$$

The solution of the control input for this particular problem is:

$$
u_{\text {heat }}(t)=h(0, t)\left(T_{1}(0, t)-\alpha T_{2}(0, t)\right)
$$

We can vary the value of the variable $b$, and observe its effect on $u(t)$. This corresponds physically to changing the height or the width of the weir located at the downstream end of the canal. Figure 3 shows the effect of varying $b$ on the control input $u(t)$.

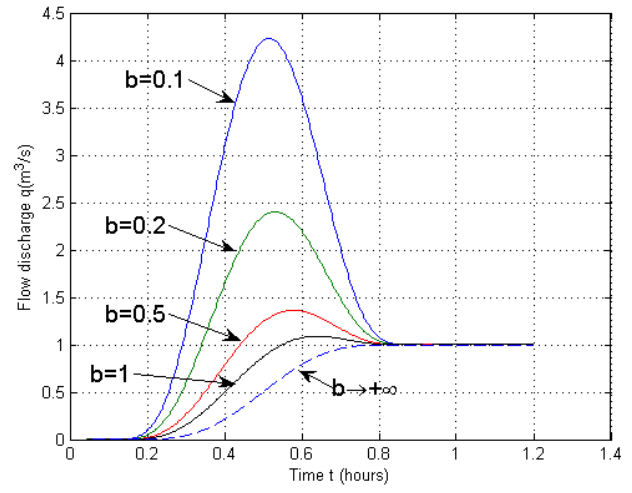

Fig. 3. Effect of varying $b$ on the upstream discharge or control input $u(t)$.

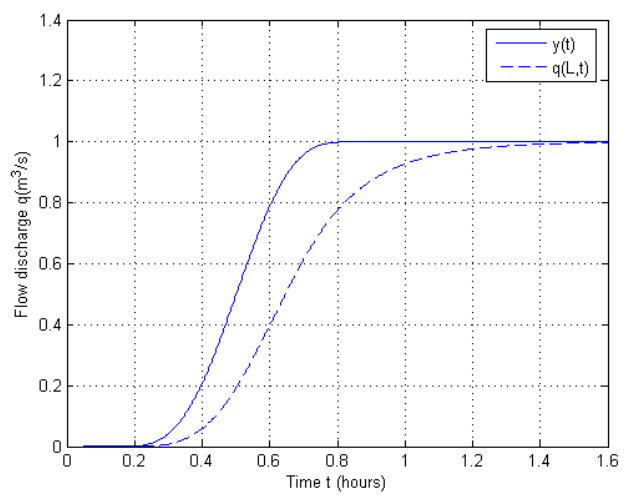

Fig. 4. Consequence of neglecting the boundary conditions in calculating the upstream discharge. The desired downstream discharge is $y(t)$, and the downstream discharge calculated by solving the Hayami model with $b=$ $1 \mathrm{~m}^{2} / \mathrm{s}$ and control input of equation (37) is $q(L, t)$.

We can see that by increasing the value of $b$, the function of $u(t)$ for our problem tend to converge to $u_{\text {heat }}(t)$ described by equation (37). This can be seen directly by studying the limit of equation (34) as $b \rightarrow \infty$ which would result in equation (37). Substituting $\kappa=\frac{B_{0}}{b} \frac{\alpha^{2}}{\beta^{2}}-\alpha$ into equation (34), we obtain:

$$
u(t)=u_{\text {heat }}(t)+u_{\mathrm{b}}(t)
$$

where

$$
u_{\mathrm{b}}(t)=h(0, t) \frac{B_{0}}{b}\left(\frac{\alpha^{2}}{\beta^{2}} T_{2}(0, t)-T_{3}(0, t)\right)
$$

As $b \rightarrow \infty$, the boundary effect will be negligible, $u_{\mathrm{b}}(t) \rightarrow 0$, and equation (34) will tend to converge in the limit to equation (37), $u(t) \rightarrow u_{\text {heat }}(t)$.

If we were to use the controller in equation (37) to control our problem with $b=1 \mathrm{~m}^{2} / \mathrm{s}$, we would obtain the results shown in figure 4.

The effect can be seen in the transition which takes approximately 1.6 hours instead of 1 hour. This shows the considerable importance of boundary conditions on the transition periods. It is therefore very important to take into account the 
boundary conditions in the open-loop control design to ensure a scheduled water distribution.

\section{B. Saint-Venant Model Simulation}

In many cases, controlling the Saint-Venant equations directly is impractical because of the need of all the geometry of the canal and the Saint-Venant parameters defined in section II-A. For this reason we have used a simplification of the model to arrive to the Hayami equation which requires only two parameters, $C_{0}$ and $D_{0}$. In this section we show numerically that a calibrated Hayami model would provide us with an open-loop control law that steers the Saint-Venant equation solution at $x=L$ or the flow discharge at the weir to the desired discharge fairly accurately. For the purpose of the simulation we will use SIC, a computer program developed by Cemagref [12] to simulate the upstream discharge and the measurement discharge at the downstream. SIC solves the full nonlinear Saint-Venant equations using a finite difference scheme (Preissmann scheme).

1) Hayami Model Identification: The purpose of model identification is to identify the parameters $C_{0}, D_{0}$ and $b$ corresponding to the Hayami model and its boundary condition that would best describe the real flow governed by the SaintVenant equations. This is done with an upstream discharge in a form of step input, the flow discharges are monitored at the upstream and downstream positions. The hydraulic identification is done classically by finding the values of $C_{0}$, $D_{0}$ and $b$ that minimize the error between the computed downstream discharge by the solution of the Crank-Nicholson scheme and the measured one. The steady flow calibration is sufficient to obtain a good dynamic model of the canal. We therefore have to solve the following optimization problem:

$$
\min _{C_{0}, D_{0}, b>0} \int_{0}^{T_{S I M}}\left|q_{S I C}(\tau)-q_{C N}\left(C_{0}, D_{0}, b, \tau\right)\right|^{2} d \tau
$$

where $q_{S I C}$ is the downstream flow generated by SIC, and $q_{C N}$ is the downstream flow generated by the CrankNicholson scheme, $T_{S I M}$ is the simulation time usually larger than the period needed to reach steady state. In our case, the identification was performed for a steady flow regime of $1.7 \mathrm{~m}^{3} / \mathrm{s}$, canal of length $L=4232 \mathrm{~m}$, and bed width $B_{0}=2 \mathrm{~m}$. This leads to the following calibrated parameters: $C_{0}=2.0160 \mathrm{~m} / \mathrm{s}, D_{0}=1517.4 \mathrm{~m}^{2} / \mathrm{s}$, and $b=0.4303 \mathrm{~m}^{2} / \mathrm{s}$.

2) Saint-Venant Control: The experimental canal we would like to simulate has the same properties as the one we have used for identification in the previous section. We are interested in raising the flow at the downstream from $2.5 \mathrm{~m}^{3} / \mathrm{s}$ to $3.5 \mathrm{~m}^{3} / \mathrm{s}$ in 4 hours. Setting the variables in section IV-A to $q_{1}=1 \mathrm{~m}^{3} / \mathrm{s}, T=14400 \mathrm{sec}$, and $\sigma=2$ will define the downstream profile $y(t)$. The control input or the discharge at the upstream can be calculated and the results are shown in figure 5 .

We notice that we have achieved the desired flow by solving the full nonlinear Saint-Venant equations. This shows that the Hayami model is practical for the design of open-loop control when the corresponding parameters are identified.

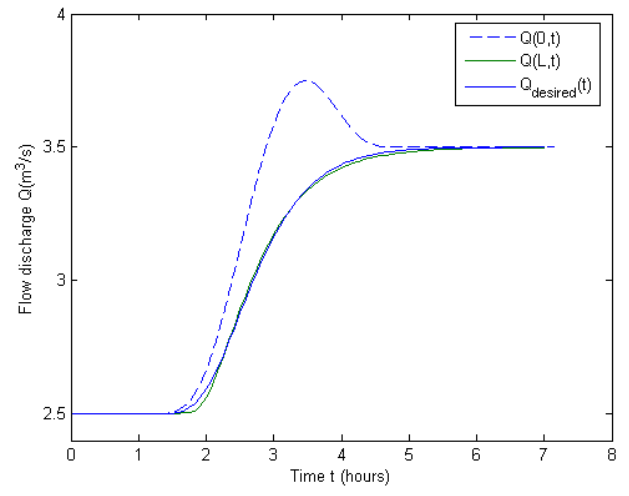

Fig. 5. Results of the implementation of our controller on the full nonlinear Saint-Venant equations. The desired downstream discharge is $Q_{\text {desidred }}(t)=$ $Q_{0}+y(t)$, the downstream discharge calculated by solving the Saint-Venant equations in SIC is $Q(L, t)=Q_{0}+q(L, t)$, and the upstream discharge of the canal is $Q(0, t)=Q_{0}+u(t)$ where $u(t)$ is calculated using the Hayami model open-loop controller. The nominal flow in the canal is $Q_{0}=2.5 \mathrm{~m}^{3} / \mathrm{s}$.

\section{CONCLUSiON}

This article introduces a new method to design an open-loop control for river flow control. The open loop control law is derived in two ways leading to identical analytical expressions.

The controller is obtained as an infinite series in terms of the desired downstream discharge flow. We have given sufficient conditions on the downstream profiles to ensure convergence. The effect of the boundary condition is also investigated and compared to previous studied ones such as the heat equation.

The simulations show satisfactory results for controlling the full Saint-Venant equation. Hydraulic engineers have shown that Saint-Venant equations can accurately model large scale open-channels. This means our methodology allows us to design open-loop controllers for large-scale system that could be cascaded and used to control a network of canals with complicated geometry. This will enable us to implement our method of open-loop controller design to Gignac Canal in Montpellier, France and further validate our results, which will be done in October 2008 with the seasonal opening of the canal for experimental purposes.

\section{ACKNOWLEDGMENT}

This article is written when Xavier Litrico was Visiting Scholar at the Civil and Environmental Engineering Department of UC Berkeley, with Prof. Alexandre Bayen. The authors want to thank Charles-Antoine Robelin for his initial work on this topic, and conversations which led to these results. Nicolas Petit is gratefully acknowledged for his help in identifying the proper work in flatness which was used in the present article. Funding support of Cemagref and the FranceBerkeley Fund is also acknowledged.

\section{REFERENCES}

[1] A. J. C. Barré de Saint-Venant. Théorie du mouvement non-permanent des eaux avec application aux crues des rivières à l'introduction des marées dans leur lit. Comptes rendus à l'Académie des Sciences, 73:148154, 237-240, 1871. 
[2] E. Bautista and A.J. Clemmens. Response of ASCE Task Committee Test Cases to Open-Loop Control Measures. Journal of Irrigation and Drainage Engineering, 125(4):179-188, 1999.

[3] E. Bautista, A.J. Clemmens, and T. Strelkoff. Comparison of Numerical Procedures for Gate Stroking. Journal of Irrigation and Drainage Engineering, 123(2):129-136, 1997.

[4] J.M. Coron, B. D’Andrea-Novel, and G. Bastin. A Lyapunov approach to control irrigation canals modeled by Saint-Venant equations. Proceedings of European Control Conference, Karlsruhe, 1999.

[5] W. Dunbar, N. Petit, P. Rouchon, and P. Martin. Motion planning for a nonlinear stefan problem. ESAIM: Control, Optimisation and Calculus of Variations, 9:275-296, 2003.

[6] M. Fliess, J.L. Lévine, P. Martin, and P. Rouchon. Flatness and defect of non-linear systems: introductory theory and examples. International Journal of Control, 61(6):1327-1361, 1995.

[7] M. Fliess, P. Martin, N. Petit, and P. Rouchon. Active signal restoration for the telegraph equation. Proceedings of the 38th IEEE Conference on Decision and Control, Phoenix, 2:1107-1111, 1999.

[8] S. Hayami. On the propagation of flood waves. Bulletin of the Disaster Prevention Institute, 1:1-16, 1951.

[9] M. Krstic. Personal communication. UC Berkeley, October 2007.

[10] B. Laroche, P. Martin, and P. Rouchon. Motion planning for a class of partial differential equations with boundary control. Proceedings of the 37th IEEE Conference on Decision and Control, Tampa, 3:3494-3497, 1998.

[11] X. Litrico. Nonlinear Diffusive Wave Modeling and Identification of Open Channels. Journal of Hydraulic Engineering, 127(4):313-320, 2001.

[12] X. Litrico, V. Fromion, J.P. Baume, C. Arranja, and M. Rijo. Experimental validation of a methodology to control irrigation canals based on Saint-Venant equations. Control Engineering Practice, 13(11):14251437, 2005.

[13] X. Litrico, V. Fromion, and G. Scorletti. Robust feedforward boundary control of hyperbolic conservation laws. Networks and Heterogeneous Media, 2(4):715-729, 2007.

[14] X. Litrico and D. Georges. Robust continuous-time and discrete-time flow control of a dam-river system: (I) modelling. Applied mathematical modelling, 23(11):809-827, 1999.

[15] A.F. Lynch and J. Rudolph. Flatness-based boundary control of a nonlinear parabolic equation modelling a tubular reactor. Nonlinear control in the year 2000, London, Lecture Notes in Control and Information Sciences 259:45-54, 2000.

[16] R. Moussa and C. Bocquillon. Criteria for the choice of flood-routing methods in natural channels. Journal of Hydrology, 186:1-30, 1996.

[17] N. Petit, Y. Creff, P. Rouchon, and P. CAS-ENSMP. Motion planning for two classes of nonlinear systems with delays depending on the control. Proceedings of the 37th IEEE Conference on Decision and Control, Tampa, 1:1007-1011, 1998.

[18] N. Petit and P. Rouchon. Flatness of Heavy Chain Systems. SIAM Journal on Control and Optimization, 40 (2):475-495, 2001.

[19] A.D. Polyanin. Handbook of Linear Partial Differential Equations for Engineers and Scientists. Chapman \& Hall/CRC, London, 2002.

[20] J. Rudolph. Planning trajectories for a class of linear partial differential equations: An introduction. Sciences et Technologies de l'Automatique. Electronic Journal: http://www. esta. see. asso. fr, 2004.

[21] B.F. Sanders and N.D. Katopodes. Adjoint sensitivity analysis for shallow-water wave control. Journal of Engineering Mechanics, 126(9):909-919, 2000.

[22] A. Smyshlyaev and M. Krstic. Lyapunov Adaptive Boundary Control for Parabolic PDEs with Spatially Varying Coefficients. Proceedings of the 2006 American Control Conference, Minneapolis, pages 41-48, 2006.

\section{APPENDIX}

Given a function $l(x, t), t \geq 0$, its temporal Laplace transform, noted $\mathcal{L}(l(x, t))$, or $\hat{l}(x, s)$ is given by: $\hat{l}(x, s)=$ $\int_{0}^{\infty} e^{-s t} l(x, t) d t$.

Starting from equations (7)-(11), we relate $\hat{q}(x, s)$ the Laplace transform of $q(x, t)$ to $\hat{q}(0, s)$ the Laplace transform of $q(0, t)$.
We have $\mathcal{L}\left(q_{t}(x, t)\right)=s \hat{q}(x, s)-q(x, 0), \mathcal{L}\left(q_{x}(x, t)\right)=$ $\hat{q}_{x}(x, s)$, and $\mathcal{L}\left(q_{x x}(x, t)\right)=\hat{q}_{x x}(x, s)$ with $q(x, 0)=0$. The property $q(x, 0)=0$ results from section II-B: $q(x, t)$ is defined to be the deviation from the nominal flow, and at $t=0$ the deviation should be 0 , i.e. $q(x, 0)=0$.

Equation (7) becomes:

$$
D_{0} \hat{q}_{x x}(x, s)-C_{0} \hat{q}_{x}(x, s)=s \hat{q}(x, s)
$$

This is an ordinary differential equation in the variable $x$, whose characteristic equation is $D_{0} \lambda^{2}-C_{0} \lambda-s=0$. The solution $\hat{q}(x, s)$ is of the following form:

$$
\hat{q}(x, s)=A_{1}(s) e^{\lambda_{1}(s) x}+A_{2}(s) e^{\lambda_{2}(s) x}
$$

with $\lambda_{1}(s)=\alpha-\gamma(s), \lambda_{2}(s)=\alpha+\gamma(s)$ and $\alpha=\frac{C_{0}}{2 D_{0}}$, $\beta=\frac{1}{\sqrt{D_{0}}}, \gamma(s)=\beta \sqrt{s+\frac{\alpha^{2}}{\beta^{2}}}$ as defined in sections III and III-B.

Substituting equation (39) into equation (8) leads to:

$$
\hat{z}(x, s)=-\frac{1}{B_{0} s}\left(A_{1}(s) \lambda_{1}(s) e^{\lambda_{1}(s) x}+A_{2}(s) \lambda_{2}(s) e^{\lambda_{2}(s) x}\right)
$$

Boundary conditions of equations (9) and (11) read as:

$$
\begin{array}{ccl}
x=0 & A_{1}(s)+A_{2}(s) & =\hat{u}(s) \\
x=L & \hat{q}(L, s) & =b \hat{z}(L, s)
\end{array}
$$

Equation (41) gives:

$$
\begin{aligned}
A_{1}(s) e^{\lambda_{1}(s) L}+A_{2}(s) e^{\lambda_{2}(s) L}= & -\frac{b}{B_{0} s}\left(A_{1}(s) \lambda_{1}(s) e^{\lambda_{1}(s) L}\right. \\
& \left.+A_{2}(s) \lambda_{2}(s) e^{\lambda_{2}(s) L}\right)
\end{aligned}
$$

which, using equation (40), leads to:

$$
A_{1}(s)=\frac{\hat{u}(s)\left(1+(\alpha+\gamma(s)) \frac{b}{B_{0} s}\right) e^{\gamma(s) L}}{D(s)}
$$

and

$$
A_{2}(s)=\frac{\hat{u}(s)\left(1+(\alpha-\gamma(s)) \frac{b}{B_{0} s}\right) e^{\gamma(s) L}}{D(s)}
$$

where

$$
\begin{aligned}
D(s)= & \frac{b}{B_{0} s}\left((\alpha+\gamma(s)) e^{\gamma(s) L}-(\alpha-\gamma(s)) e^{-\gamma(s) L}\right) \\
& +e^{\gamma(s) L}-e^{-\gamma(s) L} .
\end{aligned}
$$

Then, we obtain:

$$
\hat{q}(x, s)=F(x, s) \hat{u}(s)
$$

where

$$
\begin{gathered}
F(x, s)=\frac{P(x, s)}{Q(s)} \\
P(x, s)=e^{\alpha x}\left(\left(B_{0} s+\alpha b\right) \sinh (\gamma(s)(L-x))\right. \\
+\gamma(s) b \cosh (\gamma(s)(L-x))),
\end{gathered}
$$

and $Q(s)=\left(B_{0} s+\alpha b\right) \sinh (\gamma(s) L)+\gamma(s) b \cosh (\gamma(s) L)$. 